\title{
VALORACIÓN NUTRICIONAL DE LAS PERSONAS ADULTAS MAYORES DE ACUERDO AL AJUSTE DE TABLAS ESTANDARZADAS DE CENAPRECE EN LA ZONA URBANA DE GUERRERO, MÉXICO
}

NUTRTIONALASSESSMENT OFOLDERADULTSACCORDING TOTHEADJUSTMENTOF CENAPRECESTANDARDIZED

\section{TABLES INTHEURBAN AREAOFGUERRERO, MEXICO}

\section{Torres Castañón Mirna Eréndira ${ }^{1}$ Carreón Gómez Juan Manuel ${ }^{1}$ Bernal Mendoza Lorena Inés ${ }^{1}$ Reyna} Ávila Leticia 1

1 Escuela Superior de Enfermería No.1, de la Universidad Autónoma de Guerrero

Citation: Torres Castañón Mirna Eréndira, Carreón Gómez Juan Manuel, Bernal Mendoza Lorena Inés, Reyna Ávila Leticia. (2017) Valoración nutricional de las personas adultas mayores de acuerdo al ajuste de tablas estandarizadas de CENAPRECE en la zona urbana de Guerrero, México, Revista de Salud Pública y Nutrición, 16(2), 11-18.

Editor: Esteban G. Ramos Peña, Dr. CS., Universidad Autónoma de Nuevo león, Facultad de Salud Pública, Monterrey Nuevo León, México.

Copyright: 02017 Torres Castañon ME et al. This is an open-access article distributed under the terms of Creative Commons Attribution License [CC BY-ND 4.0], which permits unrestricted use, distribution, and reproduction in any medium, provided the original author and source are credited.

Competing interests: The authors have declared that no competing interests exist.

DOI: https://doi.org/10.29105/respyn16.2-2

Email: crysle81@hotmail.com 


\title{
VALORACIÓN NUTRICIONAL DE LAS PERSONAS ADULTAS MAYORES DE ACUERDO AL AJUSTE DE TABLAS ESTANDARIZADAS DE CENAPRECE EN LA ZONA URBANA DE GUERRERO, MÉXICO
}

\author{
Torres Castañón Mirna Eréndira ${ }^{1}$ Carreón Gómez Juan Manuel ${ }^{1}$ Bernal Mendoza Lorena Inés ${ }^{1}$ Reyna \\ Ávila Leticia ${ }^{1}$
}

1 Escuela Superior de Enfermería No.1, de la Universidad Autónoma de Guerrero

\begin{abstract}
RESUMEN
Introducción: Los principales tipos de bebidas que contribuyen con el mayor aporte energético en los escolares son las bebidas azucaradas, sin embargo, su ingesta es mayor a la recomendada y sin tomar en cuenta la del agua simple al día. Estos hábitos pueden contribuir a ganancia de peso ponderal y a padecer enfermedades crónicas. Objetivo: Ejecutar un plan de orientación nutricional sobre el consumo de bebidas en escolares de educación primaria. Métodos: Estudio descriptivo con 56 escolares de educación primaria. Se realizó en tres fases, primero se realizó antropometría, se determinó el conocimiento y frecuencia de consumo de bebidas, después cinco sesiones de orientación nutricional y finalmente se reevaluó la primera fase; se utilizó estadística descriptiva, para el procesamiento se utilizó SPSS v 21. Resultados: 56 niños del estudio, $51.8 \%$ eran mujeres, $48.2 \%$ hombres, en edad promedio de 8.1 años DE. $0.908,25 \%$ aprobó el cuestionario de conocimientos previo a las sesiones, $57 \%$ tomaban agua natural, el resto eran bebidas azucaradas, que consumían hasta más de 5 veces a la semana; posterior a la orientación nutricional el $32 \%$ incrementaron conocimiento $(p=0.007), 76.5 \%$ tomaban agua natural. Conclusiones: Se muestra una ejecución positiva del plan de orientación nutricional, se incrementó el conocimiento, sin embargo, debido a la influencia familiar sobre los hábitos alimenticios hacia los escolares no se encontró una destacada disminución en las bebidas azucaradas. Se sugiere llevar a cabo una intervención junto con los padres de familia en un periodo y muestra mayor para incrementar efectividad.
\end{abstract}

Palabras Clave: Adulto mayor, Sobrepeso y Obesidad, Envejecimiento.

\section{ABSTRACT}

Introduction: The UN considers senior adult (PAM) the 60 years for developing countries like Mexico. Nutrition plays an important role in life expectancy. The eating habits of the MAP are often inadequate. The metabolism slows down over the years, and energy requirements are lower, causing overweight and obesity $(S / O)$ reaching epidemic proportions worldwide; 2.8 million people die from it each year, and complications from chronic noncommunicable diseases. For the PAM in Mexico, ENSANUT 2012, reported on average S / O 64.25\%,33.85\% normal and 1.92\% underweight for both sexes. In Guerrero the S / O of $9.6 \%$ for both sexes. The survey reports only the state prevalence of anemia in adults aged 60 years or older, or $23.2 \%$, higher than that reported at the national level (16.5\%). Objective: To assess the nutritional status of MAP according to BMI according to the adjustment Of standardized tables of the National Center for Preventive Programs and Disease Control (CENAPRECE), as well as waist-hip circumference and associated factors. Methods: An analytical cross-sectional study, carried out in 1731 PAM of the urban area of Guerrero, random sample, selected by conglomerate. Instrument: WHO / PAHO SABE survey. Results: The prevalence of S / O was $38.19 \%$ and 19.41 of low weight according to BMI, waist measurement indicated $65.81 \%$ with risk and waist-hip ratio $76.64 \%$ high risk. Conclusions: Associated factors were age, sex, academic level, consumption of dairy products and water glasses.

Key words: Older adult, Overweight and Obesity, Aging. 


\section{Introducción}

La organización mundial de la salud, define a las personas de 60 a 74 años como edad avanzada, de 75 a 90 ancianas y mayores de 90 años son grandes viejos.

En América Latina y el Caribe en 2016, las personas de 60 años o más representan el $11,5 \%$ de la población. Se prevé que en 2030 la cifra incremente y que en 2050 constituiría el $26 \%$ de la población. (CEPAL \& ECLAC, 2016). El Consejo Nacional de Población (CONAPO), prevé que para el 2030 Guerrero se ubicara en el lugar 19 en el proceso de envejecimiento poblacional del país.

En relación a los problemas nutricionales en los adultos mayores se subraya que los cambios en el estilo de vida y la urbanización han influido en el proceso denominado "transición nutricional", el cual acompaña el proceso de transición epidemiológica y demográfica. (Acosta, Carrizo, \& Torres, 2015)

En los últimos años, las personas mayores se han convertido en una población vulnerable, rodeadas de un sinnúmero de circunstancias como las enfermedades crónicas no transmisibles, la pérdida de la capacidad funcional, el sedentarismo, la exclusión social y la disminución de la participación en el rol económico y la comunidad, lo cual afecta la calidad de vida.

A pesar de que el proceso de envejecimiento es inevitable, los desórdenes y la problemática que en general enfrenta esta población se pueden contrarrestar debido a que están influenciados por factores socioculturales y por el estilo de vida, entre ellos la alimentación y la actividad física. (Rodriguez Daza, 2011)

El proceso de envejecimiento, trae significativos cambios corporales, y la medición de la composición corporal de la población Adulta Mayor es fundamental en la evaluación del estado nutricional. La malnutrición puede tener múltiples manifestaciones de acuerdo al consumo deficiente o excesivo.

La prevalencia de malnutrición en los adultos mayores va del 4 al $10 \%$ en los que viven en su domicilio. La pérdida de peso, en sí misma no causa problemas de salud, pero hace que las personas mayores sean más vulnerables a la desnutrición y por lo tanto, a enfermar. La pérdida significativa de peso relativa al tiempo se define como pérdida aproximadamente de $2 \mathrm{~kg}$. Es un desequilibrio negativo entre el aporte de uno o más nutrientes al organismo y las necesidades de esos nutrientes, que produce una alteración en la forma $\mathrm{o}$ el funcionamiento del cuerpo.

La obesidad es una enfermedad crónica de origen multifactorial, en cuyo desarrollo están implicados determinantes genéticos y ambientales. Se manifiesta por una alteración en la composición corporal, provocando un aumento del compartimento graso. En la mayoría de los casos, el aumento de los depósitos de tejido adiposo va acompañado de un aumento del peso corporal, dando lugar a un aumento considerable del riesgo de aparición de comorbilidades que afectan la calidad y esperanza de vida.

El sobrepeso en si no es una enfermedad, pero si una condición que predispone al desarrollo de enfermedades tales como diabetes e hipertensión lo cual influye negativamente sobre enfermedades cardiovasculares (Jürschik, 2012). La OCDE reporta que entre 2000 y 2012, las tasas de sobrepeso u obesidad aumentaron de $62 \%$ a $71 \%$ y constituyen un importante factor de riesgo de defunción, con una mortalidad de alrededor de 3 millones de adultos al año. Para las personas adultos mayor (PAM) de México, en 2012, ENSANUT reporto en promedio de Sobrepeso y Obesidad (S/O), obesidad de $64.25 \%$, $33.85 \%$ normal y bajo peso un $1.92 \%$ para ambos sexos; en el sexo masculino el S/O fue de $61.93 \%$, peso normal $36 \%$ y bajo peso un $2.1 \%$; en el sexo femenino el S/O fue de $66.57 \%$, el peso normal de $31.7 \%$ y bajo peso $1.73 \%$. (Gutiérrez JP, 2012). La ENSANUT en 2012 reporto que en Guerrero la prevalencia de S/O fue de $9.6 \%$ (31.1 mil habitantes) en ambos sexos, en el femenino un $11.5 \%$ (19.7 mil habitantes) $\mathrm{y}$ en el masculino $7.4 \%$ (11.4 mil habitantes).

La prevalencia estatal de anemia en los adultos de 60 años o más fue de $23.2 \%$, la prevalencia fue mayor a la reportada en el ámbito nacional (16.5\%). Es bien sabido que la anemia durante la vida adulta disminuye considerablemente la productividad y la calidad de vida, hecho que repercute en las pérdidas económicas de las naciones. En las PAM, la anemia 
disminuye la capacidad para mantener niveles adecuados de actividad física y la movilidad en general, lo que hace a este grupo de edad más vulnerable.

Los factores asociados a los problemas nutricionales reportados en Guerrero por ENSANUT son que el $13.2 \%$ de los habitantes eran inactivos, $8.7 \%$ moderadamente activos, es decir, realizaban el mínimo de Actividad Física sugerido por la OMS, y $78.0 \%$ activos. (INSP, 2013)

Existen numerosos métodos, de mayor o menor complejidad, para estimar la composición corporal y realizar la evaluación nutricional; no obstante, el enfoque antropométrico continúa siendo la vía alternativa elegida para la mayoría de las investigaciones, no solo porque resulta de fácil acceso y aplicable a todas las personas, sino por lo inocuo, lo confiable y lo poco costoso del método. (Fernández Díaz, Martínez Fuentes, Díaz Sánchez, \& Xonia, 2005)

El objetivo del estudio fue: Valorar el estado nutricional de las Personas Adultas Mayores según IMC de acuerdo al ajuste de tablas estandarizadas de CENAPRECE, además de circunferencia de cintura, cintura- cadera y factores asociados.

\begin{abstract}
Material y Métodos
Se efectuó un estudio transversal analítico, con una muestra de 1731 personas adultas mayores de 60 años y más, de ambos sexos, con residencia mayor a 5 años en la zona urbana de Guerrero, que desearon participar con la firma del consentimiento informado o que contara con un informante sustituto en caso de que no pudieran dar la información directa. El muestreo fue aleatorio por conglomerados en cuatro etapas, en la primera etapa se seleccionaron 21 AGEBS $^{1}$ de la ciudad, en forma proporcional al grado de marginación, clasificada en 4 estratos: muy alta, alta, media y baja junto a la muy baja. Segunda etapa: de cada AGEBs se seleccionaron en forma aleatoria 10 colonias en forma proporcional al
\end{abstract}

1. AGEB urbana es un área geográfica ocupada por un conjunto de manzanas perfectamente delimitadas por calles, avenidas, andadores o cualquier otro rasgo de fácil identificación en el terreno y cuyo uso del suelo es principalmente habitacional, tamaño de las AGEBs. Tercera etapa: de cada colonia se seleccionaron 2 manzanas en forma aleatoria. Cuarta etapa: de cada manzana se seleccionaron 10 viviendas, dando un total de 4200 viviendas visitadas.

Esto permitió superar el hecho de que $55 \%$ de las viviendas no tiene adultos mayores. La unidad de muestreo fue la vivienda y la unidad de análisis los adultos mayores de las viviendas seleccionadas. La variable dependiente fue el estado nutricional de las PAM, medido a través del índice de masa corporal (IMC), Perímetro cintura, cintura-cadera. Las variables independientes: sexo, edad, estado nutricional y actividad física. Se utilizó la Encuesta Salud, Bienestar y Envejecimiento (SABE), diseñada por la OMS/OPS, tropicalizada por la Secretaría de Salud México y Guerrero. La cuál integra secciones correspondientes a las variables sociodemográficas, Estado de salud y Antropometría.

De los métodos antropométricos, el más utilizado para evaluar el estado nutricional es el índice de masa corporal (IMC), por la sencillez de su medición, que lo hace aplicable en grandes grupos de población y su fácil interpretación. Tiene mucha importancia para la epidemiología nutricional por el riesgo que constituyen para la salud los valores extremos asociados a él. (Fernández Díaz, Martínez Fuentes, Díaz Sánchez, \& Xonia, 2005)

La clasificación actual de Obesidad propuesta por la OMS está basada en el Índice de Masa Corporal (IMC), el cual corresponde a la relación entre el peso expresado en kilos y el cuadrado de la altura, expresada en metros. De esta manera, las personas cuyo cálculo de IMC sea igual o superior a 30 $\mathrm{kg} / \mathrm{m} 2$ se consideran obesas.

Este índice es la razón entre el peso (expresado en kilogramo) y la talla al cuadrado (expresada en metro) (P/T.2) Basándose en datos de morbilidad y mortalidad se ha llegado a establecer puntos de corte o valores críticos que delimitan la "normalidad" de los valores que denotan "pesos bajos" y posiblemente o ciertamente malnutrición por defecto, y los “pesos

industrial, de servicios, comercial, etcétera, y sólo son asignadas al interior de las zonas urbanas que son aquellas con población mayor o igual a 2,500 habitantes y en las cabeceras municipales. 
altos" y posiblemente o ciertamente malnutrición por exceso. (SSA, NOM-043-SSA2, 2012).

La OPS clasifica la valoración nutricional de las Personas Adultas Mayores de acuerdo al Índice de Masa Corporal (IMC), como Delgadez < 23.0, Normal > 23 a $<28$, Sobrepeso > 28 a $<32$ y Obesidad > 32. (OPS, 2002).

El perímetro de cintura se recomienda como el indicador antropométrico más práctico y sencillo para evaluar la grasa abdominal en adultos. La OMS sugiere que la medición debe tomarse $2 \mathrm{~cm}$ por debajo del ombligo, aunque puede dificultarse en personas obesas. En términos generales, circunferencias mayores a $100 \mathrm{~cm}$ se consideran riesgosas. En mujeres cuya circunferencia de cintura $>88 \mathrm{~cm}$ y en hombres con circunferencia $>$ $102 \mathrm{~cm}$ (Hombres: $\leq 102$ Normal y $>102 \mathrm{~cm}$ sobrepeso/obesidad; Mujeres: $\leq 88 \mathrm{~cm}$ Normal y $>$ $88 \mathrm{~cm}$ sobrepeso/obesidad).

La circunferencia de cintura y de cadera se expresa con el cociente cintura/cadera. La circunferencia de la cintura es indicador de tejido adiposo en la cintura y en el área abdominal; la circunferencia de cadera es un indicador de tejido adiposo que esta sobre los glúteos y la cadera, por lo tanto el cociente provee un índice de distribución de adiposidad relativa en los adultos; cuando más alto sea el cociente, mayor será la proporción de adiposidad abdominal. La clasificación de la valoración nutricional de las Personas Adultas Mayores de acuerdo a la medición cintura/cadera en hombres se considera $<0.95$ muy bajo riesgo, entre 0.96- 0.99 bajo riesgo, $>1$ alto riesgo; en mujeres se considera $<0.80$ Muy bajo riesgo, entre 0.81-0.84 bajo riesgo y $>0.85$ alto riesgo. (SSA, Evaluación y Seguimiento Nutricional del Adulto Mayor en el Primer Nivel de Atención., 2014).

Los datos se capturaron en el software Punto Penn y se trasladaron a los programas Excel y Stata V 11. La gestión de datos fue a partir de la descripción univariado, el análisis crudo bivariado se realizó calculando la RM, su intervalo de confianza 95\%, valor de "p" de la posición de la distribución " $Z$ ", con punto de corte igual o menor a 0.05 . Por último se realizó un modelo multivariado ajustado por edad y sexo.

\section{Resultados}

Participaron 1731 PAM, de los cuales $60.60 \%$ fueron Mujeres y $39.40 \%$ Hombres. La media de edad fue de $71.32 \pm 8.44$ (1DS), rango de edad de 60 a 109; De acuerdo al Nivel Académico se encontró que el $42.81 \%$ no cuentan con estudios, mientras que el $57.20 \%$ cuentan con alguna formación académica.

Referente a los hábitos alimenticios de las PAM, el $64.09 \%$ realiza dos comidas al día; el $82.99 \%$ consume lácteos al día; el $98.43 \%$ consume de leguminosas una vez a la semana, $64.42 \%$ consume de carnes tres veces por semana, $86.59 \%$ consume de verduras y frutas dos veces al día, $53.31 \%$ consume de 3 a 5 vasos de agua al día. En cuanto a la actividad física el $77.78 \%$ no realiza. (Tabla 1 )

\begin{tabular}{|c|c|c|c|c|c|c|}
\hline & \multicolumn{2}{|c|}{ Mujeres } & \multicolumn{2}{|c|}{ Hombres } & \multicolumn{2}{|c|}{ Total } \\
\hline & No. & $(\boldsymbol{x})$ & No. & $(x)$ & No. & $(\mathbf{3})$ \\
\hline \multicolumn{7}{|l|}{ Edad } \\
\hline 60 a 64 & 264 & 25.2 & 176 & 25.8 & 440 & 25.4 \\
\hline 65 a 69 & 212 & 20.2 & 155 & 22.7 & 367 & 21.2 \\
\hline 70 a 74 & 221 & 21.1 & 127 & 18.6 & 348 & 20.1 \\
\hline 75 a 79 & 162 & 15.4 & 118 & 17.3 & 280 & 16.2 \\
\hline 80 a 84 & 97 & 9.3 & 60 & 8.8 & 157 & 9.1 \\
\hline 85 y mas & 93 & 8.9 & 46 & 6.7 & 139 & 8.0 \\
\hline \multicolumn{7}{|l|}{ Nivel académico } \\
\hline Sin Estudios & 439 & 41.9 & 302 & 44.3 & 741 & 42.8 \\
\hline Nivel Básico & 396 & 37.8 & 234 & 34.3 & 630 & 36.4 \\
\hline $\begin{array}{l}\text { Nivel Medio y } \\
\text { Superior }\end{array}$ & 214 & 20.4 & 146 & 21.4 & 360 & 20.8 \\
\hline \multicolumn{7}{|c|}{ Numero de Comidas completas al dia } \\
\hline Una Comida & 18 & 1.7 & 6 & 0.9 & 24 & 1.4 \\
\hline Dos Comidas & 649 & 62.3 & 452 & 66.9 & 1,101 & 64.1 \\
\hline Tres o mas & 375 & 36.0 & 218 & 32.3 & 593 & 34.5 \\
\hline \multicolumn{7}{|l|}{ Lácteos al día } \\
\hline Si Consume & 855 & 82.1 & 570 & 84.3 & 1,425 & 83.0 \\
\hline No Consume & 186 & 17.9 & 106 & 15.7 & 292 & 17.0 \\
\hline \multicolumn{7}{|c|}{ Consumo de leguminosas una vez a la semana } \\
\hline Si & 1.029 & 98.8 & 662 & 97.9 & 1,691 & 98.4 \\
\hline No & 13 & 1.3 & 14 & 2.1 & 27 & 1.6 \\
\hline \multicolumn{7}{|c|}{ Consumo de carnes tres veces por semana } \\
\hline Si & 644 & 63.3 & 437 & 66.2 & 1,081 & 64.4 \\
\hline No & 374 & 36.7 & 223 & 33.8 & 597 & 35.6 \\
\hline \multicolumn{7}{|c|}{ Consumo de Verduras y frutas dos veces al dia } \\
\hline Si & 899 & 86.4 & 586 & 86.9 & 1,485 & 86.6 \\
\hline No & 142 & 13.6 & 88 & 13.1 & 230 & 13.4 \\
\hline \multicolumn{7}{|l|}{ Vasos de agua } \\
\hline Menos de 3 vasos & 194 & 18.7 & 117 & 17.4 & 311 & 18.2 \\
\hline De 3 a 5 vasos & 553 & 53.4 & 358 & 53.1 & 911 & 53.3 \\
\hline Más de 5 vasos & 288 & 27.8 & 199 & 29.5 & 487 & 28.5 \\
\hline \multicolumn{7}{|l|}{ Actividad fisica } \\
\hline Si & 245 & 23.6 & 136 & 20.2 & 381 & 22.2 \\
\hline No & 795 & 76.1 & 539 & 79.9 & 1,334 & 77.8 \\
\hline
\end{tabular}


De acuerdo al IMC, el S/O presenta una prevalencia de $38.19 \%$; los hombres presentan $40.76 \%$ y las mujeres $36.51 \%$; por rango de edad el grupo más afectado fue de 60 a 64 años con un $25.42 \%$. De acuerdo al IMC las personas con S/O que no hacen actividad física representan el $22.22 \%$. Considerando las tres categorías (muy bajo, bajo y alto riesgo) de la escala de clasificación de riesgo de la OMS para el perímetro cintura-cadera; se encontró que el $76.64 \%$ de las PAM se ubican en riesgo alto, por sexo el $92.73 \%$ de las mujeres tienen riesgo alto contra $51.84 \%$ de los hombres. De acuerdo a la medición del perímetro Cintura, el $65.81 \%$ presenta riesgo, por sexo se encontró que $65.36 \%$ de las mujeres tiene riesgo y en hombres $66.52 \%$. (Tabla 2)

Tabla 2. Valoración nutricional de las PAM

\begin{tabular}{|c|c|c|c|c|c|c|}
\hline & \multicolumn{2}{|c|}{ Mujeres } & \multicolumn{2}{|c|}{ Hombres } & \multicolumn{2}{|c|}{ Total } \\
\hline & No. & $(\%)$ & No. & $(\%)$ & No. & (\%) \\
\hline \multicolumn{7}{|l|}{ IMC } \\
\hline Bajo & 211 & 20.1 & 125 & 18.3 & 336 & 19.4 \\
\hline Normal & 455 & 43.4 & 279 & 40.9 & 734 & 42.4 \\
\hline syo & 383 & 36.5 & 278 & 40.8 & 661 & 38.2 \\
\hline \multicolumn{7}{|l|}{ Cintura } \\
\hline Sin Riesgo & 361 & 34.6 & 227 & 33.5 & 588 & 34.2 \\
\hline Con Riesgo & 681 & 65.4 & 451 & 66.5 & 1132 & 65.8 \\
\hline \multicolumn{7}{|c|}{ Cintura/Cadera } \\
\hline Muy Вајо & 38 & 3.6 & 175 & 25.8 & 213 & 12.4 \\
\hline Bajo & 38 & 3.6 & 152 & 22.4 & 190 & 11.0 \\
\hline Alto & 970 & 92.1 & 352 & 51.8 & 1322 & 76.6 \\
\hline
\end{tabular}

Los factores asociados al S/O mediante la medición de IMC fue la edad, el consumo de lácteos al día, consumo de vasos de agua al día. Los factores asociados en las PAM con riesgo de $\mathrm{S} / \mathrm{O}$ mediante la medición de cintura fueron la edad, el nivel académico, y el consumo de vasos de agua al día. $\mathrm{El}$ factor asociado al riesgo alto de S/O en las PAM mediante la medición de cintura cadera fue el sexo. (Tabla 3)
Tabla 3. Factores asociados al Sobrepeso y Obesidad de las PAM ${ }^{8}$

\begin{tabular}{|c|c|c|c|}
\hline Factor & $\begin{array}{c}\text { IMC } \\
\text { Cons } 5 / 0 \\
\text { RM }\end{array}$ & $\begin{array}{c}\text { Cintura } \\
\text { Con Riesgo } \\
\text { RM }\end{array}$ & $\begin{array}{c}\text { Cintura/Cadera } \\
\text { Riesgo Alto } \\
\text { RM }\end{array}$ \\
\hline Sexo & 1.2 & 1.05 & $0.10^{*}$ \\
\hline Edad & $0.94^{\circ}$ & $0.95^{\circ}$ & 1.00 \\
\hline Nivel académico & 1.03 & $1.10^{\circ}$ & 1.10 \\
\hline Numero de Comidas al dia & 0.89 & 1.19 & 1.22 \\
\hline Lácteos al día & $0.76^{* *}$ & 0.80 & 0.92 \\
\hline Consumo de leguminosas & 0.68 & 1.22 & 0.48 \\
\hline Consumo e carnes & 1.02 & 0.93 & 1.04 \\
\hline Verduras y frutas & 1.07 & 0.89 & 0.78 \\
\hline Vasos de agua & $1.34^{\circ}$ & $1.30^{*}$ & 1.15 \\
\hline Actividad fisica & 0.94 & 0.88 & 0.93 \\
\hline
\end{tabular}

8 Anślisis bivariado. $000.01 . \cdots 00.05$

Los factores asociados al bajo peso mediante la medición de IMC fue la edad, el consumo de lácteos al día, consumo de carne, y consumo de vasos de agua al día. Los factores asociados en las PAM sin riesgo mediante la medición de cintura fueron la edad, el nivel académico y el consumo de vasos de agua al día. El factor asociado muy bajo peso en las PAM mediante la medición de cintura cadera fue el sexo. (Tabla 4)

\begin{tabular}{|c|c|c|c|}
\hline actor & $\begin{array}{c}\text { IMC } \\
\text { Con bajo peso } \\
\text { RM }\end{array}$ & $\begin{array}{l}\text { Cinture } \\
\text { Sin rieseo } \\
\text { RM }\end{array}$ & $\begin{array}{c}\text { Cintura/Cadera } \\
\text { Muy bajo } \\
\text { RM }\end{array}$ \\
\hline exo & 0.89 & 1.05 & $9.21^{*}$ \\
\hline dad & $1.06^{\circ}$ & $0.96^{\circ}$ & 0.99 \\
\hline Vivel académico & 0.92 & $1.11 * *$ & 0.91 \\
\hline Vumero de Comidas al dia & 0.99 & 1.20 & 0.82 \\
\hline dicteos al dia & $1.74^{*}$ & 0.80 & 1.08 \\
\hline Consumo de leguminosas & 0.51 & 1.23 & 2.06 \\
\hline Consumo de cames & $1.20^{\circ}$ & 0.93 & 0.96 \\
\hline Verduras y frutas & 1.03 & 0.89 & 1.28 \\
\hline Vasos de agua & $0.63^{\circ}$ & $1.37^{*}$ & 0.87 \\
\hline Actividad fisica & 1.10 & 0.80 & 1.18 \\
\hline
\end{tabular}

A través del análisis multivariado se obtuvo la asociación de los siguientes factores: el S/O a través de la medición de IMC se asoció con la edad, actividad física, consumo de vasos de agua, consumo de verduras y frutas y percepción de la alimentación por las PAM. A través de la medición de Cintura se asoció con edad, consumo de vasos de agua, y percepción de la alimentación. Y a través de la medición de cintura cadera se asoció el sexo. (Tabla 5) 
Tabla 5. Sobrepeso y obesidad" a través de la medición de IMC y factores asociados (Anślisis multivariado)

\begin{tabular}{|c|c|}
\hline Medición y factores & RM \\
\hline \multicolumn{2}{|l|}{ IMC } \\
\hline Edad $=$ & 0.95 \\
\hline Sexo & 1.15 \\
\hline Actividad física* ${ }^{*}$ & 1.29 \\
\hline Consumo de vasos agua al dia $*$ & 1.27 \\
\hline Consumo de verduras y frutas" & 1.39 \\
\hline Percepción de alimentación" & 0.85 \\
\hline \multicolumn{2}{|l|}{ Cintura } \\
\hline Edad". & 1.04 \\
\hline $\operatorname{sex} 0$ & 0.94 \\
\hline Consumo de vasos agua al dí. & 0.82 \\
\hline Percepción de alimentación* & 0.78 \\
\hline Cintura Cadera & \\
\hline $\operatorname{sex} 0$ & 0.10 \\
\hline
\end{tabular}

A través del análisis multivariado se obtuvo la asociación de los siguientes factores: el bajo peso a través de la medición de IMC se asoció con la edad, lácteos al día, consumo de carnes y consumo de vasos de agua. A través de la medición de Cintura se asoció con edad, nivel académico y consumo de vasos de agua. Y a través de la medición de cintura cadera se asoció el sexo. (Tabla 6)

\begin{tabular}{|c|c|c|}
\hline Medición y Factores & RM & $P$ \\
\hline \multicolumn{3}{|l|}{ IMC } \\
\hline Edad"* & 1.05 & 0 \\
\hline Sexo & 0.91 & 0.489 \\
\hline Lácteos al día* & 1.42 & 0.023 \\
\hline Consumo de carnes* & 1.11 & 0.026 \\
\hline Consumo de vasos agua al dia $*$ & 0.72 & 0.001 \\
\hline \multicolumn{3}{|l|}{ Cintura } \\
\hline Edad* & 0.96 & 0 \\
\hline Sexo & 1.04 & 0.678 \\
\hline Nivel académico** & 1.19 & 0.001 \\
\hline Consumo de vasos agua al día & 1.23 & 0.006 \\
\hline \multicolumn{3}{|l|}{ Cintura Cadera } \\
\hline Sexo*" & 9.21 & 0 \\
\hline
\end{tabular}

Revista de Salud Pública y Nutrición / Vol. 16 No. 2 julio-septiembre, 2017

\section{Discusión}

La prevalencia de S/O encontrada en las PAM de la zona urbana del estado de Guerrero fue de $38.19 \%$, similar al estudio de Barquera realizado en México en el 2012 quien reporto un $38.8 \%$, estos datos difieren del estudio de ENSANUT en 2012 en Guerrero que reporto una prevalencia de $41.8 \%$, debido a que la población en esta investigación es de 60 años y más, y se evidenció que a partir de esta edad se va perdiendo mayor masa corporal.

La prevalencia de S/O de acuerdo al IMC reportado en este estudio es de $40.76 \%$ en hombres y $36.51 \%$ en mujeres, coincidiendo con el estudio de ENSANUT en Guerrero, en el cual el sexo que más prevaleció fue el masculino con $40.7 \%$ contra el $36.4 \%$ en el femenino. Los resultados difieren del estudio realizado por Barquera en el que la prevalencia fue mayor en el sexo femenino con un $37.5 \%$ que en el masculino $26.8 \%$. La diferencia en los hallazgos de este estudio radica en el uso de la tabla de Valoración Nutricional del Adulto Mayor que establece la OPS en Guía Clínica para Atención Primaria a las Personas Adultas Mayores, así mismo el rango de edad muestra también diferencias, ya que Barquera considera en su investigación a personas de 65 años y más y en este estudio se consideraron PAM a partir de los 60 años ( (Barquera, 2012).

Un estudio realizado por ENSANUT demuestra que la media de perímetro cintura-cadera de riesgo fue de $67.9 \%$ contra un $76.64 \%$ de este estudio. De igual forma en ENSANUT, el porcentaje para el sexo masculino fue de $70 \%$ mientras que en las mujeres fue el 74\%; resultados similares a este estudio con un $51.84 \%$ para el sexo masculino y $92.73 \%$ para el sexo femenino.

Referente al parámetro de Cintura en adultos mayores de más de 60 años de edad, los resultados de la presente investigación tomando a ambos sexos el porcentaje de riesgo fue de $65.81 \%$, en comparación con el estudio de ENSANUT donde el resultado fue de $60.95 \%$ del total de los participantes tomando en cuenta en los dos estudios a hombres y mujeres con el rango mayor a $90 \mathrm{~cm}$.

El estudio de Tarqui Mamani y colaboradores encontró que los factores sociodemográficos 
asociados con la delgadez en el adulto mayor fue el nivel educativo primario (OR 1,9; IC 95\%:1,3-2,9). Como factores asociados al sobrepeso el sexo femenino (OR 1,8; IC 95\%:1,4-2,1), y como factores sociodemográficos asociados con la obesidad el sexo femenino (OR 3,1; IC 95\%: 2,34,1 ), el nivel educativo primario (OR 2,4; IC 95\%: 1,5-4,0) o secundario (OR 2,0; IC 95\%: 1,2-3,4). (Tarqui Mamani , Álvarez Dongo , EspinozaOriundo, \& Gomez Guizado, 2014)

Coincidiendo con el presente estudio, en el cual se reportó que los factores asociados al S/O mediante la medición de cintura fue el nivel académico $(\mathrm{p}=0.009)$ y mediante el indicador de cintura cadera se asoció con el sexo femenino $(\mathrm{p}=0.000)$.

Los factores asociados al $\mathrm{S} / \mathrm{O}$ reportados en este estudio fueron: a través de la medición de IMC (edad, actividad física, consumo de vasos de agua al día, consumo de frutas y percepción de la alimentación por parte de las PAM), a través de la medición de cintura (edad, consumo de vasos de agua y percepción de la alimentación por parte de las PAM) y a través de la medición de cintura cadera (sexo). Los factores asociados al bajo peso: a través de la medición de IMC (edad, consumo de lácteos al día, consumo de carnes y consumo de vasos de agua al día, a través de la medición de cintura (edad, nivel académico, y consumo de vasos de agua) y a través de la medición de cintura cadera (sexo).

\section{Conclusiones:}

Los resultados del estudio, hacen necesaria la reorientación de políticas públicas y actividades que conduzcan a modificar la salud y la atención para el bienestar de las PAM.

El aporte del presente estudio es la integración de las tres mediciones para detectar el $\mathrm{S} / \mathrm{O}$, así como el bajo peso en las PAM. Por lo tanto es fundamental que en la medición de cintura y cintura cadera se realicen nuevos puntos de corte adaptados a las PAM, ya que los establecidos por la OMS son generales y podrían sobre representar el $\mathrm{S} / \mathrm{O}$ en este grupo poblacional.

\section{Agradecimientos:}

Agradecimiento a CENAPRECE, Universidad Autónoma de Guerrero, Secretaria de Salud Guerrero y a los estudiantes participantes de la unidad académica de enfermería No. 1 de la UAGro, por permitirnos trabajar en conjunto durante el desarrollo del proyecto SABE, así mismo por el apoyo y confianza brindada al grupo disciplinar.

\section{Bibliografía}

Acosta, L. D., Carrizo, E. D., \& Torres, V. E. (2015). Life conditions, nutritional and health status in elderly population. Brasileira de Geriatria e Gerontologia, 1(18), 107-118. Recuperado el 08 de Noviembre de 2016, de_https://dx.doi.org/10.1590/18099823.2015.14058

Barquera. (2012). Obesidad en adultos, los retos de la cuesta abajo. Recuperado el 2017, de http://ensanut.insp.mx/doctos/analiticos/ObesidadAdu ltos.pdf

CEPAL, \& ECLAC. (2016). Envejecimiento $e$ institucionalidad pública en América Latina y el Caribe: conceptos, metodologías y casos prácticos (Vols. 16-00435). (N. Unidas, Ed.) Chile, Santiago de Chile : CEPAL. Recuperado el 07 de Noviembre de 2016, de Disponible en: http://repositorio.cepal.org/bitstream/handle/11362/40 197/S1600435_es.pdf?sequence=1\&isAllowed=y

Fernández Díaz, I. E., Martínez Fuentes, A. J., Díaz Sánchez, M. E., \& Xonia, X. M. (2005). Evaluación nutricional antropométrica en ancianos. Rev Cubana Med Gen Integr(21), 1-2. Obtenido de www.bvs.sld.cu/revistas/mgi/vol21_1-2_05/mgi071$\underline{205 t . h t m}$

Gutiérrez JP, R.-D. J.-L.-H.-N.-M.-Á. ( 2012). Encuesta Nacional de Salud y Nutrición 2012. Resultados Nacionales. (Primera edición). (I. N. (MX), Ed.) Cuernavaca, México.

INSP. (2013). Encuesta Nacional de Salud y Nutrición 2012. Resultados por entidad federativa,. (Primera edición electrónica). Guerrero. Cuernavaca, México. Obtenido de encuestas.insp.mx

Jürschik. (SEPTIEMBRE de 2012). la valoración nutricional de los individuos mayores. Obtenido de la valoración nutricional de los individuos mayores: http://www.aanep.com/docs/Consenso-FinalEvaluacion-Nutricional.pdf 
OPS. (2002). Valoración Nutricional del Adulto Mayor. En Guía Clínica para Atención Primaria a las Personas Adultas Mayores. Washington, DC. Obtenido de file:///C:/Users/Equipo/Documents/CUERPO\%20AC ADEMICO $\% 20$ RIESGOS\%20A\%20LA\%20SALUD $\%$ 20COLECTIVA/PROYECTO\%20SABE/Guía\%20 VNA\%20Adulto\%20MayorESCALA\%20MININUTRICIONAL.pdf

Rodriguez Daza, K. D. (2011). Vejez y Envejecimiento. (p. edicion, Ed.) Grupo de Investigacion en actividad fisica y desarrollo humano(12), 42.

doi:Doc.Inv.Esc.Med.Cs.Salud

SSA. (2012). NOM-043-SSA2-2012.Servicios básicos de salud. Promoción y educación para la salud en materia alimentaria. Criterios para brindar orientación. Obtenido de NORMA Oficial Mexicana NOM-043SSA2-2012. Servicios básicos de salud. Promoción y educación para la salud en materia alimentaria.

Criterios para brindar orientación.: http://www.dof.gob.mx/nota_detalle.php?codigo $=528$ $5372 \&$ fecha $=22 / 01 / 2013$

SSA. (11 de diciembre de 2014). Evaluación y Seguimiento Nutricional del Adulto Mayor en el Primer Nivel de Atención. Seguro Social IMSS-095$08 . \quad$ Obtenido de http://www.cenetec.salud.gob.mx/interior/catalogoMa estroGPC.html

Tarqui Mamani , C., Álvarez Dongo , D., EspinozaOriundo, P., \& Gomez Guizado, G. (2014). Estado nutricional asociado a características sociodemográficas en el adulto mayor peruano. Rev Peru Med Exp Salud Publica, 31(3), 467-72. 